\title{
La procedencia, a escala local, de los inmigrantes extranjeros en España a finales del siglo $X X$
}

\author{
Ma Pilar González Yanci y $M^{\underline{a}}$ José Aguilera Arilla *
}

\begin{abstract}
RESUMEN
El articulo propone la utilización de los registros consulares, como fuente de investigación imprescindible para el estudio pormenorizado del origen $y$ destino de los inmigrantes extranjeros en nuestro país. Dicha fuente es la única que recoge, a escala local, el lugar de nacimiento y el de procedencia, según el último domicilio del pais de origen, asi como su lugar de residencia en el país de destino. Del estudio de las fuentes consulares de la República Checa, Eslovaquia y Bulgaria, se obtiene la idea general de que existe una mayor procedencia urbana de los inmigrantes, a pesar de no ser paises con alto porcentaje de población urbana. También el estudio comparativo de los lugares de nacimiento y de procedencia, permiten conocer la existencia de movimientos

internos previos, de relativa importancia. Una vez en España, se

\section{ABSTRACT}

This article proposes the use of consular records as a source of investigation essential to the detailed study of the origin and destination of foreign immigrants in our country. The mentioned source is the only one that provides, on a local stage, the place of birth and origin, according to the last address in the native country, as well as the place of residence in the destination country. By studying consular sources from the Czech Republic, Slovakia and Bulgaria, we obtain the general idea that immigrants come mainly from urban areas, although those countries do not have a high percentage of urban population. Besides, the comparative study of the places of birth and origin makes possible to know the existence of previous internal migrations of relative importance. Once in Spain,
\end{abstract}

* U.N.E.D. Madrid. 
distribuyen por las regiones más prósperas, la Capital, Levante y las Islas, y se detecta una clara preferencia por las zonas turísticas con mejor clima. they spread on the most thriving areas: the capital, Levant and the Islands, and they show a clear preference for tourist areas with better climate.

\section{INTRODUCCIÓN}

Los estudios acerca de los inmigrantes extranjeros ponen su acento, por regla general, en algunas características de los mismos, como son, las sociodemográficas: edad, sexo, estado civil, formación, profesión, composición familiar y su reunificación; las de su inserción en la sociedad que los acoge, dificultades que sufren los inmigrantes: integración/adaptación, segunda generación, aprendizaje del idioma del país de acogida, cultura del nuevo país, racismo/xenofobia, etc.; la legislación que afecta a las migraciones: asilo/refugio, leyes de extranjería, instituciones y organizaciones que se ocupan de ellos: civiles, religiosas, estatales, ONG; incluso de su distribución espacial dentro del país de acogida, y si acuden a zonas rurales o urbanas. Sin embargo, no hay apenas estudios de los lugares de procedencia, fuera del ámbito nacional, de los inmigrantes extranjeros. Quizá desde el punto de vista de otros investigadores la escala nacional, o incluso de gran conjunto regional, pueda ser suficiente, pero, para un geógrafo, la procedencia local, o provincial, resulta muy interesante, sobre todo para poder comparar con otros momentos históricos, $u$ otros comportamientos diferentes entre medio rural o urbano, de otros países de parecido ámbito económico.

En nuestra línea de investigación, venimos analizando la realidad de la nueva fase inmigratoria que se está produciendo en España, con especial atención puesta en la relación entre las características socioeconómicas y su establecimiento en el país (características de las áreas sociales en las que se instalan) así como en analizar las particularidades de determinados grupos de inmigrantes (del Este, cubanos, del Tercer Mundo, del Magreb, etc.) y en la percepción que los españoles tienen de este nuevo fenómeno en nuestro país. A lo largo de estos trabajos hemos hecho hincapié en el análisis de origen y destino. Resulta interesante, por otro lado, despejar la incógnita de la procedencia, si es del ámbito rural o del urbano, conocer el tamaño de las localidades de origen, saber si se trata de áreas industriales o agrarias, en crisis o prósperas, de si es su primer movimiento espacial o si, por el contrario, han sido ya emigrantes dentro de su propio país. 
En la actualidad, parece evidente que el principal destino de los inmigrantes, tanto en migraciones interiores como exteriores es, en gran medida, urbano. Sin olvidar que también acuden a determinadas zonas agrarias, o mineras, inmigrantes extranjeros, sobre todo desde el mundo subdesarrollado. La procedencia, durante mucho tiempo, ha sido eminentemente rural, también tanto en los movimientos interiores, como en los internacionales. Sin embargo ¿conocemos, en estos momentos, de qué ámbitos proceden los inmigrantes? En España, las estadísticas no permiten averiguar este dato.

\section{LA FUENTE CONSULAR}

En la búsqueda de obtener información al respecto, en el caso de los inmigrantes extranjeros en España, nos hemos dirigido a los Servicios Consulares de las Embajadas con sede en nuestro pais, como informantes de primera mano, encontrando que constituyen una importante fuente, aunque como algunas otras, plagada de dificultades, entre las que se pueden destacar las siguientes:

- Difícil acceso a su utilización, ya que no existe homogeneidad entre los diferentes consulados (mientras unos no permiten utilizarla, otros dan todo tipo de facilidades).

- En general, estos datos no están informatizados y muchas veces no están actualizados.

- La fuente es selectiva, puesto que no recoge a todos los inmigrantes, sólo se registran los que acuden a su embajada por algún motivo personal.

- A veces, existen verdaderas dificultades en el idioma, alfabeto (árabe, cirílico...), etc.

A pesar de todas estas dificultades es, hoy por hoy, la mejor fuente que hemos conseguido y un valioso complemento para las restantes, de uso común.

Las fichas consulares, aunque con diferencias de unos países a otros, proporcionan una información sociodemográfica de relativo interés, por cuanto no difieren fundamentalmente de la que se obtiene por medio de otros cauces (Censo, Estadísticas de permisos de trabajo, Anuario de migraciones,etc.). Dicha información se refiere a edad, sexo, estado civil y, en algún caso, profesión. Además, proporciona datos relativos a la procedencia y lugar de nacimiento de estos inmigrantes, así como a su destino 
en España, en ambos casos a escala local, información que no puede obtenerse de ninguna otra fuente de las anteriormente citadas. En estos datos radica su principal interés.

Vamos, en este artículo, a mostrar algunos ejemplos de utilización de esta fuente, tomados de uno de los flujos que hoy reviste un cierto interés, por su novedad, en nuestro país. Se trata de algunas de las nacionalidades de la Europa del Este, que, como es sabido, desde los cambios acaecidos tras la caída del muro de Berlín, constituyen un importante polo emigratorio, y en las últimas tendencias ya inmigratorio, en algunos casos, (SOPEMI 1997). Suponen cifras significativas en el caso que vamos a utilizar: en el de la República Checa y Eslovaquia totalizan 613 personas, cuando el censo de España de 1991 daba 447 extranjeros nacidos en Checoslovaquia y las Estadísticas de permisos de trabajo en vigor a 31 de diciembre de 1995 daban 449. En el caso de la República de Bulgaria los registrados alcanzan una cifra de 1.352 personas, frente a los 239 del Censo, los 533 de las Estadísticas de permisos de trabajo y los 863 del Anuario de migraciones de 1995.

\section{EL CASO CHECO.}

Con un cierto equilibrio por sexo, aunque predominan las mujeres (52\% frente al $48 \%$ de varones) son, en general, jóvenes (el $53 \%$ entre 20 y 34 años) con muy pocos efectivos de menores de 20 y mayores de 60 ( 8 y $10 \%$ repectivamente). Una población, por tanto, típicamente inmigrante, en la que lo más destacable, como anomalía, es el predominio de mujeres, sobre todo en el grupo de 25 a 29 años (Figura 1). La tendencia a no declarar el estado civil hace que este dato resulte irrelevante, no obstante, entre los que lo declaran, se aprecia un absoluto predominio de casados $(70 \%)$.

Lo realmente interesante es la información que se puede obtener relativa a la procedencia. La mayoria de los checos llegan a España desde el mismo lugar en que nacieron (el 59\%), en cambio, el 34\% (el resto no lo declara), proceden de un lugar distinto al de su nacimiento, lo que indica, al menos, una migración previa. Dicha migración fue interna en la mayoría de los casos, ya que sólo en dos ocasiones el punto de partida es de fuera de la República Checa y además de países muy lejanos (Chile y Sudáfrica). Algunos otros casos de procedentes y ciudadanos checos manifiestan un lugar de nacimiento fuera de aquellas fronteras, en Italia, Moscú o Dakar, lo que también ındıca que hubo migración anterior de sus padres y que, tras acudir al país de origen, realizan una nueva migración. 

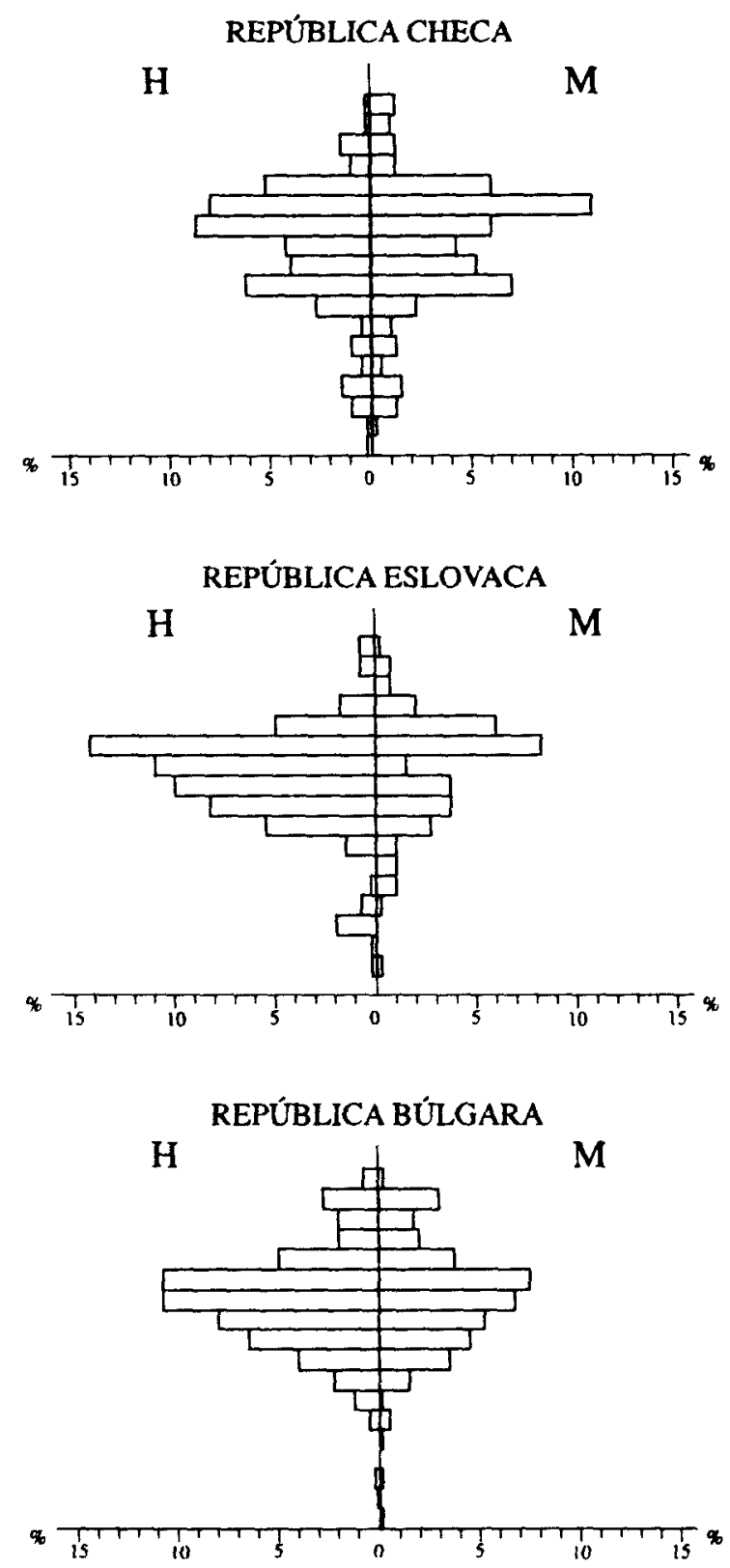

Figura 1. Pirámides de población de los inmigrantes checos, eslovacos y búlgaros en España, a partir de los datos del registro consular (E. P.). 
Los casos de personas que emigraron, o retornaron desde el exterior de la República Checa, son sólo testimoniales por su reducido número. La mıgración interna se ha producido entre multitud de lugares, pero con un claro predominio de destino en Praga, la capital del Estado, el $38 \%$ de los que migraron interiormente acudieron a la capital, porcentaje que se eleva a casi la mitad si se considera la llegada a las principales ciudades (Brno, Usti nad Laben, Ostrava y PIzen) lo que puede ser indicativo de un proceso previo de éxodo rural.

No obstante, es mucho más numeroso el grupo de personas que han venido a España directamente desde el lugar en el que nacieron y donde tenían su último domicilio, destacando que esto ocurría en el $60 \%$ de los casos y de ellos, cerca de la mitad eran de Praga, proporción que se eleva a dos tercios del total si se considera a los de las mayores ciudades (Praga, Brno, Usti nad Laben, Plzen, Ostrava, Hradek Kralove) claro indicativo de que en este país se había ya realizado, desde antiguo un movimiento hacia las grandes ciudades. La procedencia actual de los checos que vienen a España resulta muy expresiva (Figura 2). El $41 \%$ del total vienen de la capital nacional, Praga, y el $22 \%$ del conjunto de las principales ciudades (Brno, capital de Moravia del Sur, Plzen, capital de Bohemia Occidental, Ostrava, Capital de Moravia Norte y Usti nad Laben, capital de Bohemia Norte) totalizando un $63 \%$ de las grandes ciudades (Cuadro 1). El $36 \%$ restante se reparte, la mitad procedente de ciudades de tamaño medio y el resto de ciudades pequeñas y pueblos. Resulta así que el $78.4 \%$ proceden de áreas urbanas y principalmente de ciudades industriales, con dos grandes focos: el más occidental con Praga, Plzen y Ustí nad Laben y el más oriental, con la región de Ostrava, IOstrava, Kárvina, Frydek Mistek, Novijicin) y BrnoZlin.

Cuadro 1. Población y porcentaje de inmigrantes, en España, de las grandes ciudades checas.

\begin{tabular}{lrc}
\hline CIUDAD & POBLACIÓN EN 1994 & $\begin{array}{c}\% \text { DE INMIGRANTES } \\
\text { DE ESTE ORIGEN }\end{array}$ \\
\hline PRAGA & 1.217 .000 & 41.2 \\
BRNO & 390.000 & 5.6 \\
OSTRAVA & 327.000 & 3.6 \\
KÁRVINA & 286.000 & 2.6 \\
OLOMUC & 225.000 & 2.0 \\
ZLIN & 198.000 & 1.6 \\
PLZEN & 172.000 & 4.3 \\
\hline
\end{tabular}

Fuente: Statesman Year book (1997) y Registro Consular (e.p.). 
Este dato resulta destacable, dado que la República Checa posee una población urbana de tan sólo un $65.4 \%$. Además, se da una clara coincidencia entre los lugares de procedencia y las áreas más industriales del país, más castigadas por la crisis, que ha afectado a las antiguas regiones industriales de Europa y de manera muy especial a las de los países del Este, tras el proceso de desmantelamiento de las antiguas estructuras en la órbita del comunismo, que han provocado la aparición del paro, a pesar de que las cifras, en 1996, eran de tan sólo un 3\% (entre 1993 y 1996 los desempleados pasaron de 93.380 a 159.000). La actividad industrial fue importante en esta región desde el pasado. En la época del Imperio, las regiones de Bohemia y Moravia ya constituian, junto a la cuenca de Viena, los focos principales, que con los Habsburgo suministraban material de equipamiento al Imperio y hasta exportaban. Con la Primera República Checoslovaca (1919-38) hubo un esfuerzo de industrialización, con importante producción de mecánica especializada, a la que se orientó la región checa. Después de 1946 el esfuerzo se hizo para desarrollar una industria pesada, básica para suministrar a las zonas menos desarrolladas de Europa Central. En la actualidad ha recuperado parte de la antigua tradición de producción de calidad y constituye uno de los países más industrializados de los del Este de Europa. Destacando las industrias de la región de Ostrava-Zlín (siderurgia, química, cemento...) y de Praga-Plzen (mecánica, alimentaria, vidrio, etc.)

En el Cuarto Informe periódico realizado por la Comisión de las Comunidades Europeas, en 1991, se llamaba la atención sobre la situación de los Seis (así se denominaba al conjunto de Polonia, Yugoslavia, Rumanía, Checoslovaquia, Hungría y Bulgaria) en cuanto a los problemas que se consideraba eran probables motivos de expulsión para la población potencialmente emigrante. Destacaban el elevado grado de industrialización en estos paises, y el alto porcentaje de empleo industrial, pero con un sector industrial obsoleto y en crisis, por estar configurado de acuerdo con la anterior situación económica planificada y centralizada y precisar de una transformación, erizada de dificultades, hacia la economía de mercado. También se destacaban los elevados porcentajes de empleo en el sector agrario, con problemas igualmente de adecuación a la economía de mercado y el elevadísimo porcentaje de empleo en un sector terciario dominado por los servicios del Gobierno Central. Todo ello ayuda a explicar, en primer lugar, la migración interna previa que se detecta (paso de personas empleadas en el campo a ciudades industriales y capitales) y en segundo lugar la procedencia final desde las mayores ciudades castigadas por la crisis de su industria y la pérdida de empleo en los servicios estatales. (COMISION. C.E. 1991). 


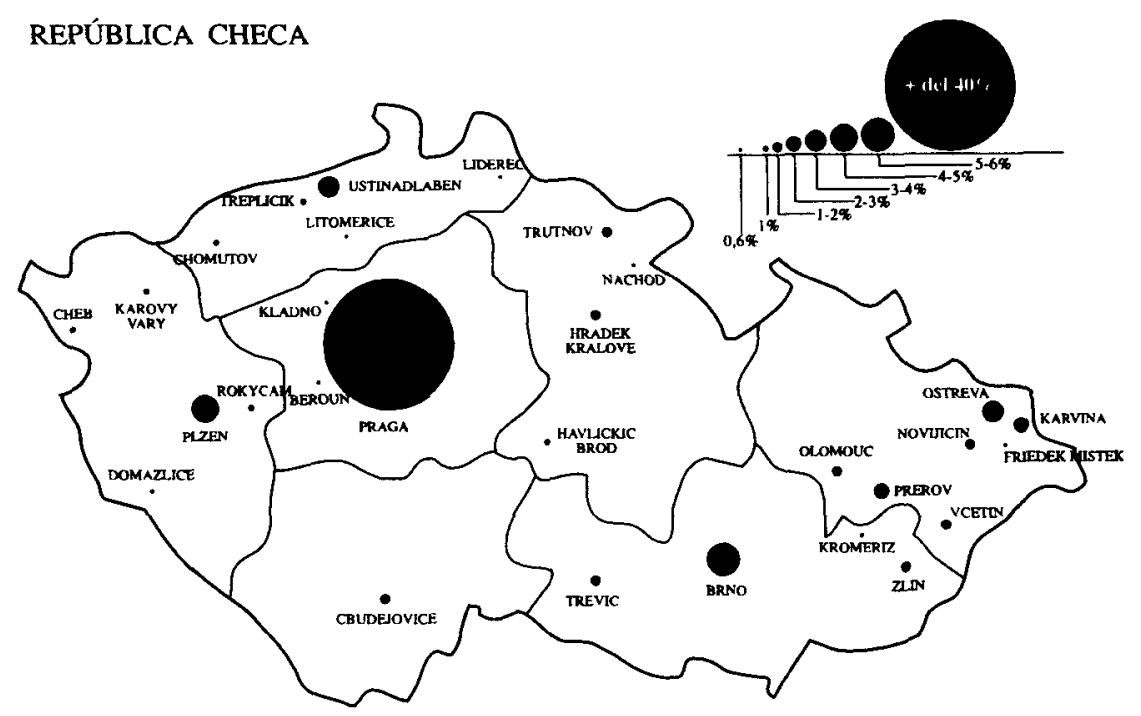

Figura 2. Procedencia de los emigrantes checos en España. Fuente: elaboración propia a partir de los datos consulares.

Una vez en España, también a partir de la fuente consular, se puede analizar la distribución espacial con información más rica que la que facilitan las fuentes estadísticas oficiales, dado que, en el caso de las fichas consulares, se hace indicación expresa de la localidad a la que acuden, a escala mucho más detallada que en las anteriores, donde se refieren tan sólo a comunidades o provincias.

En principio no coincide la distribución espacial en España con la que se obtiene de la fuente de los permisos de trabajo, en buena parte, porque en esta fuente no diferencian las nacionalidades checa y eslovaca '.

Los hechos más destacables de la distribución espacial son:

- Acuden a las grandes ciudades (Madrid el 28\% y Barcelona el 16\%) a las regiones costeras del Este (Gerona, Tarragona, Castellón, Valencia, Alicante y Murcia suman el $22 \%$, al que si se añade el de Barcelona asciende al $38 \%$ ) y a las islas (Baleares y Canarias reciben a un $13 \%$ ).

1 Tras diversos avatares en el pasado, de 1969 a 1992 Checoslovaquia fue un estado constituído por dos repúblicas federadas. El 31 de diciembre de 1992 se dividió en dos estados denominados República Checa y República Eslovaca. Los ciudadanos pudieron, en una primera etapa, elegir nacionalidad, incluso mantener la doble nacionalidad en Eslovaquia. Desde 1994 se les asigna nacionalidad según el lugar de nacimiento. 
- Aunque en proporción muy pequeña, hay alguna presencia en la mayoría de las provincias, (sólo 15 no tienen ningún inmigrante checo). Hay que destacar que en algún caso acuden a zonas mineras de Asturias, aunque en proporción muy inferior a sus vecinos los eslovacos.

- También es destacable que, por destinos, hay bastante equilibrio entre sexos, con excepciones como Murcia y Asturias, donde sólo hay varones y las islas, donde predominan las mujeres.

- Resulta muy interesante la tendencia a residir en poblaciones distintas de la capital. En Madrid, el $35 \%$ de los que viven en la Comunidad lo hacen en localidades fuera de la capital, la mayoría del entorno metropolitano, pero también en algunos pueblos de la Sierra. En Barcelona también siguen esta pauta de distribución el $18 \%$ y en las restantes provincias casi la totalidad viven en pueblos, especialmente los que tienen un desarrollo de actividades turísticas (Marbella, Torremolinos, Benidorm, Almuñecar, Lloret de Mar, etc.).

\section{EL CASO ESLOVACO.}

Los datos de los ciudadanos de origen eslovaco resultan semejantes a los de los checos, en cuanto al predominio de jóvenes (el $40 \%$ entre 25 y 35 años), con especial acento en el peldaño de 25 a 29 , junto a un marcado déficit de niños y ancianos, como corresponde a una población netamente inmigrante, que, además, se ha producido desde tiempos recientes (el $87 \%$ de los eclovacos registrados en el consulado habían llegado a España entre 1990 y 1995). En cambio, en este caso, el desequilibrio entre los sexos se decanta a favor de los varones, que casi suponen el $57 \%$ del total (Figura 1).

Aunque son pocos los que lo declaran, en este caso tenemos el dato añadido de los que expresan su profesión (19\%), resultando muy llamativo el hecho de que la mayoría son mineros que trabajaban en las minas de Ostrava (República Checa) y se trasladan a las de Asturias. Los restantes que lo declaran destacan por su alto nivel de formación: músicos, actores, universitarios y empresarios.

En esta información se da una coincidencia con los resultados de analizar las fuentes estadísticas españolas, donde se pone de manifiesto que los inmigrantes del Este, tienen un nivel de cualificación más elevado que los de otras procedencias (G. YANCI, $M^{a}$ P. y AGUILERA, $M^{a}$ J. y 1999).

En el caso de los eslovacos tan sólo en el 35\% conocemos el dato de su último domicilio y su lugar de nacimiento. Aunque sea un número re- 
ducido permite extraer conclusiones interesantes. Para más de la mitad $(55 \%)$ coincidían aquellos dos lugares y por tanto, parece tratarse de su primera migración. El $45 \%$ restante ha realizado, al menos, otro movimiento, que casi siempre es interno (algunos proceden de la República Checa, del tiempo en que ambos países formaban uno sólo). Otros tantos proceden de otro país, Andorra, RDA, o han nacido en otro, como Hungría u Holanda). En el resto de casos, que muestran una anterior migración interna, es de pueblos a ciudades pequeñas y medias, sin que haya un aplastante predominio de los que acuden a las mayores ciudades, como Bratislava o Kosice, que son, no obstante, el destino más buscado.

Entre los que proceden directamente de su lugar de nacimiento y que, por tanto, probablemente no habian tenido movilidad anterior, el predominio es de los que tienen un origen urbano, destacando casi la mitad que son y proceden de la capital Bratislava (Figura 3).

\section{ESLOVAQUIA}

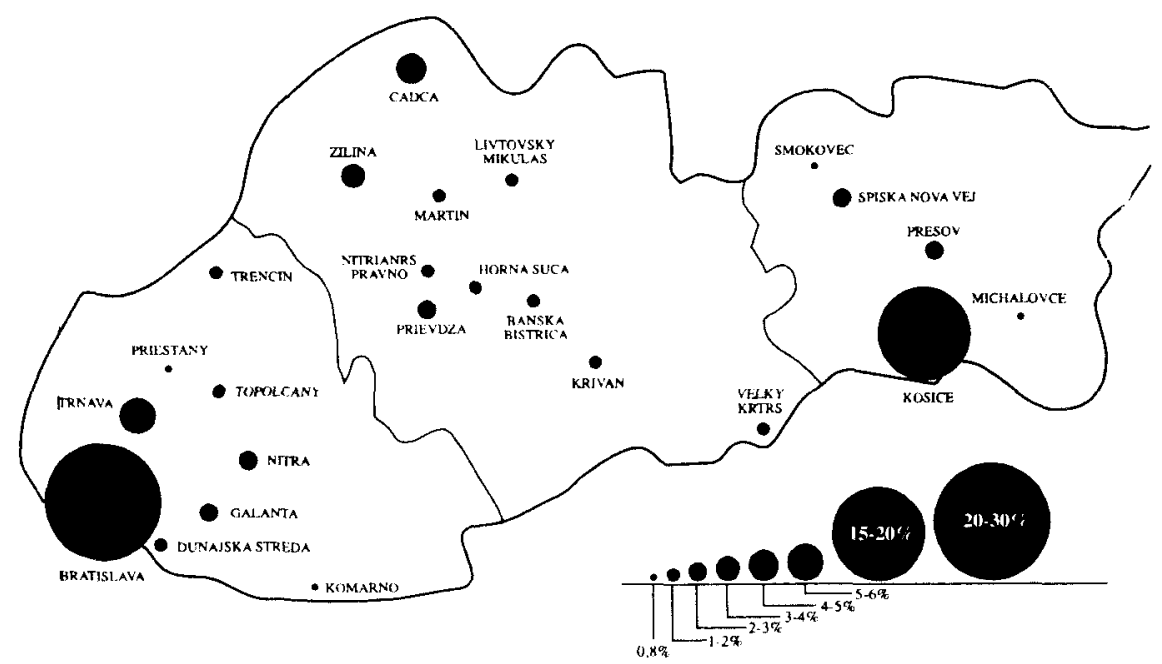

Figura 3. Procedencia de los emigrantes eslovacos en España. Fuente: elaboración propia a partir de los datos consulares.

En conjunto la procedencia es esencialmente de ciudades, pese a que, en el caso de Eslovaquia, la población urbana es de tan sólo el $58.8 \%$, y con total predominio de las principales urbes $(23 \%$ de la capital Bratislava y $15 \%$ de la siguiente ciudad en importancia, Kosice). La tercera capital regional, Banska Bystrica, sólo envía un $2 \%$, mientras que son ciudades me- 
dias, como Trnava, de las zonas más industriales, las que se reparten el $33 \%$ restante. En suma, más del $70 \%$ procede de ciudades situadas fuera de las áreas más agrícolas del país (Cuadro 2). Bratislava, Kosice, Banska Bystrica y Zilina son algunas de las principales ciudades industriales desde que, tras la segunda Guerra Mundial, se comenzó a superar la anterior situación de región subdesarrollada y sin industria que presentaba Eslovaquia, con la creación de establecimientos industriales de siderurgia y metalurgia de metales no ferrosos (región de Kosice y Banska Bystrica) y de madera, celulosa y textil en la región de Zilina, además de Bratislava, que posee sobre todo industria metalúrgica. Industrias afectadas, al igual que en el caso checo, por la crisis. Las cifras de desempleo superaban en 1996 el $13 \%$ en el pais, habiendo pasado en sólo 6 meses del 12.3 al $13.3 \%$.

Cuadro 2. Población y porcentaje de inmigrantes, en España, de las ciudades eslovacas.

\begin{tabular}{lrc}
\hline CIUDAD & POBLACIÓN EN 1993 & $\begin{array}{c}\% \text { DE INMIGRANTES } \\
\text { DE ESTE ORIGEN }\end{array}$ \\
\hline BRATISLAVA & 446.655 & 22.9 \\
KOSICE & 237.336 & 15.3 \\
NITRA & 90.866 & 2.4 \\
PRESOV & 90.069 & 2.4 \\
BANSKA BYSTRICA & 85.631 & 2.0 \\
ZILINE & 85.005 & 4.0 \\
TRNAVA & 72.244 & 5.2 \\
FRIEVDZE & 54.024 & 2.8 \\
\hline
\end{tabular}

Fuente: C. Atlante Agostini, 1997 y Registro Consular (e.p.).

El destino en España presenta la fuerte presencia de eslovacos en las minas asturianas. Declaran su destino 265 , por tanto el $92 \%$. De ellos hay $24 \%$ en Asturias, todos varones, de entre 30 y 45 años, que llegan a España en 1993. Sigue Madrid con un $21 \%$ y Barcelona con un $8 \%$. El resto de destinos, con pocos efectivos, es a varias provincias, pero con mayoria en las Islas (15.5\%), en Levante (Tarragona, Castellón, Valencia, Alicante y Murcia totalizan $10.5 \%$ ) y Málaga (10.2\%).

Al igual que en el caso de los checos, lo más significativo es la tendencia a vivir en pueblos fuera de la capital. En Madrid, por ejemplo, el $34 \%$ de los que están en la Comunidad viven en poblaciones fuera de la ciudad. 
El último informe realizado por la Comisión Europea arroja luz sobre la situación de los dos países analizados, que ayuda a explicar el mapa de origen de los emigrantes checos y eslovacos y las principales causas de expulsión. Estos países, junto a Polonia y Hungría, constituyen un conjunto denominado del acuerdo de Visegrad, que ha sido objeto de especial atención por parte de la Comisión citada. Desde 1989, al abandonarse la planificación central y producirse una espectacular transformación económica, hubo en estos países, una tremenda caída de la producción, una subida de las tasas de inflación (que alcanzaron hasta un 600\%) y un fuerte incremento del desempleo, que, de prácticamente cero, pasó a una media de $12-14 \%$ en 1993 (con notables diferencias regionales). Con ello hubo una disminución de los salarios reales, aumento de precios y un cada vez más difícil y desigual acceso de la población a la vivienda, la sanidad y la educación, en un proceso que durante un período de tiempo se preveía iría empeorando y distanciándose de las condiciones de Europa Occidental. De hecho, entre 1989 y 1992, aunque con diferencias regionales y entre países, ha sido importante el descenso del número de puestos de trabajo, especialmente agudo en las ciudades y regiones en que la producción se concentraba en una única actividad y en concreto en sectores de la energía, minería, industria pesada y de defensa. También descendieron, de forma considerable, los puestos de trabajo en el sector agrario, sobre todo en las regiones cercanas a las grandes ciudades y en el sector público, por cierre o racionalización de las empresas estatales, mientras que sólo creció el número de empleos en el sector servicios de las ciudades. Junto a esto, en el mismo período y, fundamentalmente, por el hundimiento de las empresas estatales, hubo una gran caída del PIB en todas las regiones. La falta de perspectivas, pese a la opinión inicial de que los flujos de capital acudirian al Este, han sido el detonante de salida para unos emigrantes que proceden, sobre todo, de las regiones más industriales (COMISIÓN EUROPEA,1994).

\section{EL CASO BÚLGARO}

Los búlgaros, que constituyen en el registro consular un grupo mucho más numeroso que los anteriores y que los registrados en el Censo ${ }^{2}$, presentan una diferencia entre los sexos mayor que en los casos anteriores. Aquí los varones alcanzan el $58 \%$, es decir, una proporción de 1.4 varones

2 Téngase en cuenta que los datos de los registros consulares recogen cifras más actualizadas que las estadisticas españolas, comprendiendo las considerables llegadas de los años 91-95. 
por mujer. Al incorporar la edad (Figura 1) se observa que el contingente de varones es más numeroso que el de mujeres, precisamente en las edades de mayor actividad laboral, en las que algunos grupos quinquenales masculinos superan en un $2 \%$ a los femeninos. Eso confirma el carácter netamente económico de este flujo migratorio, al igual que en los casos anteriores (G. YANCI, M.P. y AGUILERA, M.J. 1999). Respecto a su estado civil, sólo un $58 \%$ del total de los registros contienen ese dato. Entre los que lo manifiestan, existe una mayoría de casados (52.4\%). Los divorciados alcanzan un $2.9 \%$, lo que unido a un testimonial $0.4 \%$ de viudos, deja un $44.3 \%$ de solteros, proporción también bastante elevada. Los búlgaros, según lo que acabamos de exponer, parecen movilizarse tanto en familia, como individualmente $y$, tanto solteros, como casados.

A pesar de lo interesante que resultaría conocer la profesión, de esta variable sólo poseemos 95 respuestas (menos del 10\% del total). Sin embargo, debemos realzar su valor ya que esos 95 casos se concentran en los que llegan a nuestro país entre 1990 y 1991, pues con referencia a ellos, la proporción llega a suponer un $31.2 \%$ del total de registrados en esos años. En conjunto, destaca el hecho de que músicos, deportistas y artistas forman el grupo más numeroso, seguidos de becarios, estudiantes de doctorado o del CSIC, que son, junto a algunos profesionales liberales, los de mayor nivel de estudios, y con los profesionales de empresas hispano-búlgaras, los más cualificados. Todos ellos superan, con mucho, a los que declaran una profesión con menor cualificación (10\%), corroborando la existencia de un mayor nivel profesional de los primeros emigrantes del Este, que hemos puesto de relieve en otras investigaciones, aspecto que, no obstante, en la actualidad se está transformando.

Centrándonos ahora en lo que da a la fuente consular su carácter de exclusividad, es decir, en el lugar de nacimiento y el lugar del que proceden, según su última residencia en Bulgaria, se observa que también los búlgaros llegan a España procedentes, en su mayoría, de la misma ciudad en que nacieron (55.5\%). Sin embargo, se constata que existe un primer movimiento interno, al comprobar que un $29 \%$ ha cambiado su domicilio antes de la salida del país, al menos una vez. En ese movimiento, el principal foco de atracción es la capital nacional, Sofía (10\%) y también desde las aldeas, acuden a las ciudades próximas, muchas veces a las capitales regionales a las que pertenecen, y otras, a ciudades industriales, desarrolladas tras la Segunda Guerra Mundial. Esto supone un claro indicador de un proceso de éxodo rural, aunque el más importante, el que se produjo en los años 50, con los planes quinquenales, cuando más de 50.000 campesinos se convirtieron en obreros, resulta demasiado lejano en el tiempo, dadas las edades que presentan los inmigrantes búlgaros. No obs- 
tante, ha habido movimientos recientes campo-ciudad, puesto que la población urbana pasó de ser del $62 \%$ en 1983 al $70.7 \%$ diez años más tarde, y ese crecimiento no puede deberse tan sólo a un crecimiento natural que, por otra parte, es actualmente negativo en el país (-1.2 entre 1989-94). Junto con la capital Sofía, Plovdiv, capital regional de la depresión del Maritza, y Pleven, de la plataforma Danubiana, son los otros dos focos de atracción en los movimientos internos.

El movimiento migratorio previo detectado es fundamentalmente interno, pues aunque aparecen personas nacidas en otros países pertenecientes al antiguo bloque comunista, no son muy numerosas. Sin embargo, sí son significativos los nacidos en Cuba, URSS, e incluso Polonia, lo que demuestra una clara, aunque escasa, movilidad entre estos países, relacionados políticamente en el pasado, aunque su cifra sea reducida y sirva sólo como demostración de la escasa movilidad exterior en la etapa comunista.

Otro hecho que permite comprobar el movimiento interno es que, mientras las localidades en las que nacieron son 217, las que declaran como último domicilio son tan sólo 100 , aunque existe un $15 \%$ de los registrados que no informa de su último domicilio en Bulgaria y sólo un $3 \%$ que no dice donde nació.

La proporción de los que sí manifiestan su última residencia $(85 \%)$ es lo suficientemente amplia como para formarnos una idea fiel de que son más los que no han hecho un movimiento interno en su país, aparentemente al menos, aunque previamente sus padres hayan realizado el éxodo rural, que los que han emigrado dentro del mismo. De los que sí han cambiado, predomina el paso de aldeas a las ciudades principales con actividad industrial, de su provincia (voivodia), que el movimiento entre ciudades.

Bulgaria es hoy un país bastante urbanizado si lo comparamos con otros de su entorno, el $70.7 \%$ de su población de 1995 vivía en ciudades, por lo que es muy lógico que esa procedencia, la urbana, sea la más numerosa. El principal origen es la capital nacional (42.5\%), que también es la más poblada de Bulgaria (Figura 4). Si a él le sumamos las proporciones de los que proceden de las siguientes seis ciudades más pobladas Plovdiv, Varna, Ruse, Stara Zagora, Pleven y Sliven, la proporción asciende al $63.8 \%$ del total de procedencias. El porcentaje restante se reparte entre ciudades relativamente grandes, como Burgas, y otras de tamaño medio y pequeño, hasta completar un $72.8 \%$, cifra que sumada con los que no responden viene a suponer prácticamente el $90 \%$ del total, quedando un reducido $10 \%$ procedente de pequeñas ciudades, pueblos y aldeas (Cuadro 3). 


\section{BULGARIA}
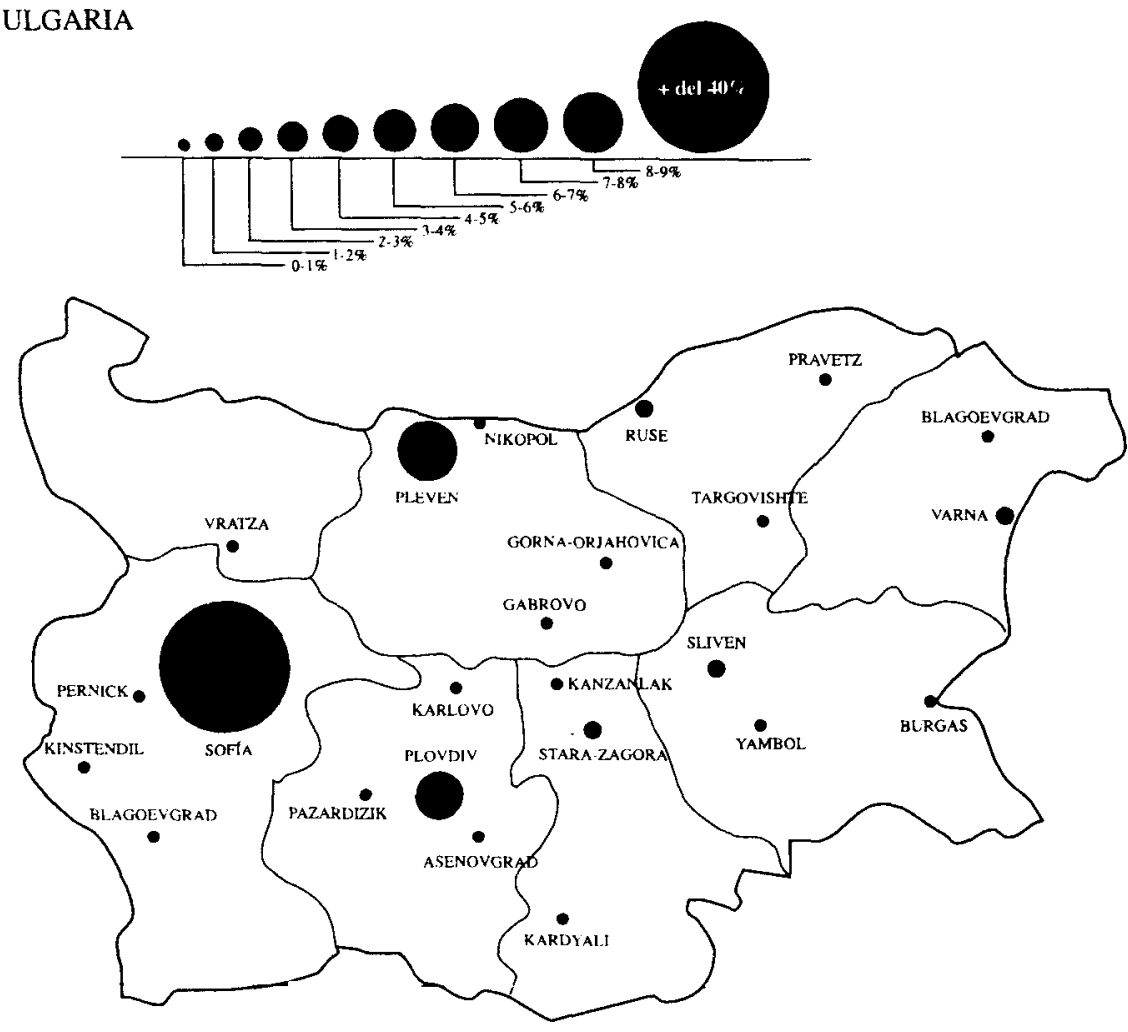

Figura 4. Procedencia de los emigrantes búlgaros en España. Fuente: elaboración propia a partir de los datos consulares.

Cuadro 3. Población y porcentaje de inmigrantes, en España, de las ciudades búlgaras.

\begin{tabular}{lrc}
\hline CIUDAD & POBLACIÓN (1992) & $\begin{array}{c}\text { \% DE INMIGRANTES } \\
\text { DE ESTE ORIGEN }\end{array}$ \\
\hline SOFÍA & 1.116 .454 & 42.5 \\
PLOVDIV & 346.330 & 6.6 \\
VARNA & 304.499 & 1.5 \\
BURGAS & 199.869 & 0.4 \\
RUSE & 168.609 & 1.6 \\
STARA-ZAGORA & 151.218 & 1.1 \\
PLEVEN & 127.945 & 9.0 \\
SLIVEN & 107.267 & 1.5 \\
\hline
\end{tabular}

Fuente: Statesman Yearbook, 1997 y Registro Consular (e.p.). 
En resumen, los focos fundamentales son los de Sofía y las capitales regionales de Plovdiv y Pleven, que coinciden con las áreas más pobladas e industrializadas. Tras la Segunda Guerra Mundial la inversión soviética introdujo la industria siderúrgica en Bulgaria. También se crearon importantes astilleros e industria de transformación, sobre todo alimentaria y de otros productos agrícolas (tabaco) por todo el país. Alrededor de las ciudades se localizaron pequeños talleres artesanales. La actividad industrial actual es bastante diversa (industrias pesadas, material ferroviario, textil, química, cuero, papel, aparatos eléctricos, materiales de construcción, etc.) además de que existe una actividad turística, de la que se benefician los servicios y la construcción. Destacan los centros de Sofía y Plovdiv, y en menor número en Pleven, ciudades que constituyen, además, importantes centros culturales, to que proporciona a sus habitantes mayores oportunidades de preparación y cualificación. Estos aspectos, que en un momento de desarrollo resultaron de atracción para la inmigración interna, en momentos difíciles, como los que están viviendo estos países, por la crisis que se ha producido con el cambio a una economía de libre mercado, en que las cifras de paro ascienden al $11.6 \%$ de la población activa, son los que favorecen la salida del país. A los citados aspectos hay que sumar la baja renta per cápita de Bulgaria (1.160 \$USA en 1994), menos de la mitad que la de Eslovaquia (2.650 \$) y un tercio de la de la República Checa (3.210 \$).

Desde el punto de vista de los propios inmigrantes búlgaros, existe un sentimiento de dispersión, e incluso de cierto aislamiento, dentro de nuestro territorio nacional, hecho que también contemplan algunos mediadores sociales, cuando declaran que este colectivo se halla muy disperso en España. Los datos obtenidos en el Consulado muestran que los búlgaros, llegados entre 1990 y 1995, se distribuyen siguiendo la pauta más generalizada entre los inmigrantes del Este, aunque no coincidente con la distribución obtenida por otras fuentes, ni para algunas otras nacionalidades. Los aspectos más destacables son:

- Frente a un gran número de residentes en la Comunidad Autónoma de Madrid (59.08\%) un 32\% se distribuyen por todas las demás.

- Aunque en algunos casos en proporciones muy pequeñas, como en el de Murcia con un $0.07 \%$, se distribuyen por todas las Comunidades Autónomas y por casi todas las provincias (sólo 9 no tienen ningún inmigrante búlgaro). Por capitales provinciales, tras el $37.2 \%$ de Madrid capital se sitúa, a mucha distancia, Barcelona (2.9\%), Valencia (1.8\%), Palma de Mallorca (1.6\%) y Málaga (1.4\%); las restantes no superan el $1 \%$.

- Un aspecto que resulta muy interesante es el gran número de ellos que manifiesta residir en municipios no capitales de provincia. Sólo en la 
Comunidad madrileña habitan en 36 poblaciones ( $11.8 \%$ del total), la mayoría metropolitanas (Alcalá de Henares $4.4 \%$, Getafe 3.3\% y Móstoles $2.1 \%$ ), pero también viven en otras de la Sierra, aunque en menor proporción. En el resto del territorio, son 25 los lugares de residencia que no son capitales, la mayoría de ellos costeros. Las causas residen probablemente en el menor precio de las viviendas, en el primero de los casos, y en el lugar donde han podido encontrar empleo con mayor facilidad, en el segundo.

\section{CONCLUSIÓN}

De las tres nacionalidades estudiadas a través de la fuente consular, como puede verse en el cuadro 4, se desprenden algunas semejanzas y diferencias en el origen y destino a escala local de sus emigrantes.

Cuadro 4. Análisis comparativo de origen y destino.

\begin{tabular}{|c|c|c|c|}
\hline & BULGARIA & REP. CHECA & ESLOVAQUIA \\
\hline$\sum_{\substack{w \\
\frac{\pi}{0}}}^{\lessgtr}$ & $\begin{array}{l}55 \% \text { coincide lugar de } \\
\text { nacimiento y procedencia. }\end{array}$ & $\begin{array}{l}59 \% \text { coincide lugar de } \\
\text { nacimiento y procedencia. }\end{array}$ & $\begin{array}{l}55 \% \text { coincide lugar de } \\
\text { nacimiento y procedencia. }\end{array}$ \\
\hline $\begin{array}{l}\frac{Z}{0} \\
\frac{1}{2} \\
\frac{1}{0} \\
\frac{1}{2} \\
\Sigma\end{array}$ & $\begin{array}{l}29 \% \text { migración interior } \\
\text { previa. }\end{array}$ & $\begin{array}{l}34 \% \text { revelan una migración } \\
\text { previa. Casi el } 40 \% \text { con } \\
\text { destino primero en la } \\
\text { capital Praga. }\end{array}$ & $\begin{array}{l}45 \% \text { han realizado } \\
\text { migración interna previa } \\
\text { con destino en ciudades } \\
\text { de tamaño medio. }\end{array}$ \\
\hline 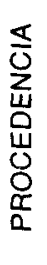 & $\begin{array}{l}42 \% \text { de la capital Sofía. } \\
20 \% \text { de las mayores } \\
\text { ciudades. }\end{array}$ & $\begin{array}{l}41 \% \text { de la capital Praga. } \\
22 \% \text { de las grandes } \\
\text { ciudades capitales } \\
\text { regionales. } \\
36 \% \text { repartido entre } \\
\text { ciudades medias con } \\
\text { implantación industrial. }\end{array}$ & $\begin{array}{l}23 \% \text { de la capital } \\
\text { Bratislava. } \\
15 \% \text { de Kosice capital } \\
\text { regional. } \\
16 \% \text { de ciudades } \\
\text { mayores. }\end{array}$ \\
\hline $\begin{array}{l}O \\
\vdots \\
\mathfrak{k} \\
\text { 岁 }\end{array}$ & $\begin{array}{l}59 \% \text { a Madrid } \\
11 \% \text { a la costa Este, } \\
\text { de Gerona a Alicante. } \\
5 \% \text { a las Islas. }\end{array}$ & $\begin{array}{l}38 \% \text { a Levante, de Gerona } \\
\text { a Murcia. } \\
28 \% \text { a Madrid } \\
13 \% \text { a las Islas. }\end{array}$ & $\begin{array}{l}24 \% \text { a la región minera } \\
\text { asturiana. } \\
21 \% \text { a Madrid } \\
28 \% \text { a la costa Este de } \\
\text { Gerona a Málaga. } \\
15 \% \text { a las Islas. }\end{array}$ \\
\hline
\end{tabular}

Fuente: Elaboración propia a partir de los datos del registro Consular. 
Hay una considerable migración interna previa a la salida al exterior. La procedencia última es predominantemente de la capital del Estado y de las grandes ciudades, especialmente con actividad industrial. Una vez en España, se distribuyen por todo el territorio, pero con diversos focos, entre los que Madrid es el principal (salvo la excepción de los mineros de Asturias) y le siguen las ciudades de la costa Mediterránea, desde Gerona hasta Málaga, y las islas, sobre todo Canarias, regiones de importante actividad turística, en todos los casos. Además, es especialmente significativo que un elevadísimo número viven en poblaciones no capitales de provincia, con marcado carácter turístico (Marbella, Torremolinos, Benidorm, etc.) y que ofrecen condiciones climáticas deseadas por los europeos del Este, dato que conocemos por fuentes orales. Así como en las áreas metropolitanas de las grandes ciudades (Madrid y Barcelona) en las que se unen factores de menores precios de las viviendas, con otros de tipo personal y hasta psicológico, como el del tamaño de sus poblaciones, que las hace más asequibles.

En definitiva, de este estudio se desprende que, al tener acceso a una fuente como la consular, se puede obtener una rica información sobre el origen y el destino de los inmigrantes, con la que podemos mejorar el conocimiento de los principales factores de expulsión y atracción. El hecho de que los inmigrantes tengan una procedencia eminentemente urbana, a pesar de ser países con tasas de urbanización relativamente bajas, y precisamente el que vengan desde las ciudades de mayor actividad industrial, indica el papel que juega en la expulsión la crisis producida en la industria de estos países. Los bajísimos salarios y el cierre de empresas por un lado, junto a las expectativas de mejorar en paises de la órbita occidental, por otro, así como el deseo de conocer lo que durante mucho tiempo les estuvo vedado, de lo que la información es mayor para los habitantes de ciudades, parecen ser las causas más directas. En el destino en España, se aprecia la tendencia a vivir en las áreas más prósperas, como es natural, que posibilitan una mayor oferta de empleo y unas condiciones de vida atractivas, por diversas razones.

No obstante, éste resulta un ejemplo limitado. Sería interesante hacerlo extensivo a otros países de distinto ámbito económico y cultural, para llegar a conclusiones más universales.

\section{BIBLIOGRAFÍA}

CHESNAIS, (1992): "La emigración de Europa Central y Oriental, tendencias e intereses en juego" En Política Exterior ${ }^{\circ} 6$ pág. 41-56.

COLE, J. (1996): Geography of the World's Major Reqions. Ed. Routledge. London and New York. 476 págs. 
La procedencia, a escala local, de los inmigrantes extranjeros en España...

COMISION DE LAS COMUNIDADES EUROPEAS, (1991): Las regiones en la década de los noventa. Cuarto Informe periódico sobre la situación y evolución socioeconómica de las regiones de la Comunidad. Luxemburgo, 114 págs.

COMISION EUROPEA, (1994): Competitividad y cohesión, las tendencias de las regiones. Quinto Informe periódico sobre la situación y la evolución socioeconómica de las regiones de la Comunidad. Oficina de Publicaciones Oficiales de las Comunidades Europeas, Luxemburgo-Bruselas. 207 págs.

CONSEJO DE EUROPA, (1994) Actividades del Consejo de Europa en el dominio de las migraciones. "La migration est-ouest en Europe» Groupe de Vienne, págs. 21-24.

EPRC, (1993): «Regional Socio-economic development in Poland, Hungary, the Czech Republic and Slovaquia». Estudio financiado por la DG XVI de la Comisión Europea.

Gonzalez-Yanci, M.P.; Aguilera ARILla, M.J. (1999):"Los países del Este, de nuevo foco de emigración de la Europa actual. Su incidencia en España." En Estudios Geográficos.

GuHLLón, M. (1989): "Refugies et immigrés d Europe de l'Est." En Revue Europpéenne des Migrations Internationals. Vol. $5 n^{\circ} 3$, págs. 133-138.

KUPIZZEWSKI (1993): $\gg$ Migration from Eastern Europe to European Community: Current and futura development". En Working Paper 94/4. School of Geography. The University of Leeds, U.K.

Nera, (1992): "Trade and Foreign Investment in the Community's regions: the impact of economic reform in Central and Eastern Europe." Regional Development Studies n-7. Estudio financiado por la DG XVI de la Comisión Europea.

SOPEMI (1997): Trends in international migration. Annual report 1996. OCDE. París. 274 págs. 